\title{
Hacia la renovación en las políticas de infancia en Chile
}

\author{
PAULA BEDREGAL MD. MPH.PhD
}

1. Profesora Asociada, Departamento de Salud Pública, Facultad de Medicina UC

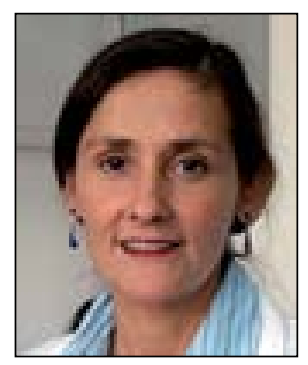

Chile ha venido adoptando diversas medidas para concentrar esfuerzos de inversión social en la infancia, las que se han traducido en iniciativas relevantes para el país y para el resto de las Américas, como son: el Sistema de Protección Integral a la Infancia Chile Crece Contigo (en adelante CCC), la promulgación de la Ley 20.545 (2011) que extiende el período posnatal a veinticuatro semanas y, la instauración de la obligatoriedad del kínder.

En salud infantil, las políticas centradas en la reducción de la mortalidad infantil la lucha contra las enfermedades infecciosas (en particular inmuno-prevenibles) y la malnutrición por déficit, han colocado a Chile en una posición de ventaja respecto del resto de Sudamérica. Para 2012, Chile muestra una mortalidad en menores de 5 años, de 9 por 1.000 , ubicándose en el sexto lugar de menor mortalidad en las Américas, y el segundo en Sudamérica (superado por Uruguay con una tasa de 7 por 1.000). En el contexto mundial, los países con menor tasa son Luxemburgo e Islandia con 2 y los países escandinavos con 3 por 1.000 . Desde 1990 la reducción en Chile de la mortalidad en menores de 5 años ha sido sostenida, cayendo un $52 \%{ }^{1}$.

Estas mejoras también se deben a las condiciones generales de vida. En Chile ha habido un incremento del Producto Interno Bruto per cápita, el que alcanzó en 2012 a US\$ 16.200. Esta cifra esconde desigualdades en la distribución de la riqueza, como lo expresa el coeficiente de GINI de 2012 con un valor de 0,55 (más cerca de 0 más equitativo) ${ }^{2}$. Por otra parte, para 2011, se observa una reducción general de la pobreza a cifras del 14\%; sin embargo, los menores de 18 años muestran cifras del $22 \%$, lo que implica que 1 de cada cuatro menores de 18 sean pobres, cifra que se eleva a 1 de cada tres en menores de 4 años ${ }^{3}$.

El análisis actual de la situación de salud de la infancia muestra un panorama diferente al del siglo pasado. En mortalidad infantil, los esfuerzos deberían continuar en el área neonatal, y en particular la prematuridad extrema, que contribuye en un $43 \%$ a la mortalidad infantil. En niños mayores, la principal causa sigue siendo las causas externas (accidentes y violencias) con $38,1 \%$, seguido de neoplasias $(13,6 \%)$ y de enfermedades del sistema nervioso (12\%). La mayor sobrevida de nuestros niños, ha hecho evidente la presencia de enfermedades crónicas de la infancia, entre éstas la malnutrición por exceso, con cifras alarmantes y crecientes de un 33\% en menores de 6 años del sector público de salud. Otra enfermedad muy prevalente y crónica, son las caries dentales, que afectan $70,4 \%$ de los niños y niñas de 6 años de edad ${ }^{4}$.

Adicionalmente múltiples estudios nos muestran que la situación de desarrollo psicosocial infantil no ha mejorado desde 1980, en que se instalan los primeros esfuerzos sistemáticos en esta materia. Las cifras de múltiples estudios siguen cercanas al 30\% de niños con

Recibido el 3 de febrero de 2014.

Correspondencia a:

Paula Bedregal

E-mail: pbedrega@med.puc.c 
desarrollo alterado (tabla 1$)^{5}$. Por otra parte, la frecuencia de rezagos y retrasos medida en el sector público de salud con EEDP y TEPSI, nos arroja cifras entre enero y octubre de 2013 de $11,6 \%$ en niños entre 7 y 59 meses de edad (DEIS-MINSAL, 2013). Esta discrepancia nos habla de la necesidad de mejorar la calidad de la pesquisa en el sector.

La prevalencia de trastornos psiquiátricos en niños y niñas entre 4 y 18 años alcanza valores de 38,3\% (EE: $2,3 \%)^{6}$; en niños menores de 5 años del sector público de salud los trastornos conductuales afectan al 30\% ${ }^{7}$. El abuso y maltrato infantil, como problema de salud y social, muestra para 2012 que el $71 \%$ de escolares chilenos declaran haber sufrido algún tipo de violencia por parte de sus padres, y un $8,7 \%$ abuso sexual ${ }^{8}$. A esto, se les suma un grupo particularmente dañado y vulnerado en sus derechos, como son los niños y niñas víctimas de explotación sexual comercial, que trabajan, que viven en situación de calle o que están en implicados en hechos delictuales.

Este perfil de sobrevivencia, cronicidad y contexto social hace evidente la emergencia del grupo de niños y niñas con necesidades especiales de atención en salud (NANEAS), y que tiene un correlato para el sector educación en los llamados niños y niñas con necesidades especiales educativas.

Estos antecedentes, colocan en evidencia la necesidad de actualizar y modificar las políticas de salud en infancia.

Mundialmente, este nuevo milenio nos interpela sobre la calidad e integralidad de las políticas de infancia. Esto, porque para lograr mantener la reducción en la mortalidad infantil y mejorar la calidad de vida de los niños, niñas y sus familias se requieren de nuevos enfoques. Tradicionalmente, el abordaje ha sido sectorial (por ejemplo: sector educación y salud por su lado) y por programas o prestaciones específicas. Sin embargo, la tendencia en la actualidad es a constituir sistemas integrados, cuyo propósito final es contribuir al bienestar de niños y niñas y sus familias, en una lógica de Protección Social a la infancia, en que cada sector social contribuye al logro 5 .

Chile fue pionero en la región con la implementación el 2007 del Subsistema Chile Crece
Contigo (Ley 20.379, de 2009), cuyo objetivo es fortalecer las prestaciones entregadas para la primera infancia (de 0 a 4 años) para favorecer el desarrollo psicosocial de niños y niñas. CCC cuenta con programas a cargo del sector salud, como es el Programa de Apoyo al Desarrollo Biopsicosocial (eje del sistema) y el Programa de Apoyo al Recién Nacido. Las evidencias actualmente disponibles de las evaluaciones de Chile Crece Contigo muestran que nos encontramos en una etapa inicial de implementación del conjunto de prestaciones y que el resultado en desarrollo psicosocial depende de la intensidad de uso de los servicios.

El modelo CCC en la región de las Américas ha sido pionero por su estructura organizacional de base local, e intersectorial. Otros países, como Colombia, con "Cero a Siempre", Ecuador, con la estrategia intersectorial de infancia; Uruguay, con "Uruguay Crece Contigo"; por ejemplo, han avanzado integrando aspectos de desarrollo psicosocial con la atención en salud tradicional, a la primera infancia, en sistemas. Los ejes de estas políticas son mejorar el acceso a prestaciones de calidad, la coordinación intersectorial, y la corresponsabilidad familiar, bajo un enfoque de derechos de la niñez.

¿Que se espera para Chile? En salud infantil, el objetivo sanitario y meta para la década 2010-2020, es la reducción del rezago infantil en un $15 \%$. A éste se le suman objetivos transversales como son aquellos en el área de enfermedades transmisibles, respiratorias agudas, problemas crónicos, y factores de riesgo de enfermedades cardiovasculares.

¿Qué se esperaría? En la línea de la Protección Social para el sector salud, se requiere de identificar garantías universales (sector público y privado) dado un enfoque de derechos. Para esto es clave la integración programática más clara entre el Programa de Salud Infantil y los otros programas destinados a infancia como el Programa de Apoyo al Desarrollo Psicosocial (de CCC), las garantías explícitas en salud (GES), en un modelo de atención de salud infantil, que ponga como eje el desarrollo humano (entendiendo como tal, los aspectos biológicos, psicológicos y sociales del desarrollo integral).

Esto implica superar modelos tradiciona- 
Tabla 1. Prevalencia de desarrollo alterado en subgrupos de población. Algunos estudios disponibles'

\begin{tabular}{|c|c|c|c|c|c|}
\hline Grupo de edad & Población & Instrumento $^{2}$ & Estudio & Año & Tasa \% \\
\hline $12-23$ meses & Nacional & $\mathrm{DIEH}$ & ENCAVI & 2006 & 31,5 \\
\hline 24-59 meses & Nacional & DIEH & ENCAVI & 2006 & 31,1 \\
\hline 3-15 meses & Jardines JUNJI & BATTELLE & Evaluación JUNJI & 2007 & 32,1 \\
\hline 24-36 meses & Medio menor & BATTELLE & Evaluación JUNJI & 2007 & 27,7 \\
\hline$<24$ meses & Nacional & EEDP & ELPI-1 & 2010 & 24,8 \\
\hline 25-72 meses & Nacional & TVIP (LENGUAJE) & ELPI-1 & 2010 & 26,1 \\
\hline 30-48 meses & $\begin{array}{l}\text { Sector público } \\
\text { salud }\end{array}$ & BATTELLE & $\begin{array}{l}\text { Evaluación efectividad del Programa de Apoyo } \\
\text { al Desarrollo Biopsicosocial. Cohorte control }\end{array}$ & 2010 & 31,8 \\
\hline 6-83 meses & Nacional & BATTELLE-2 TAMIZAJE & ELPI-2 & 2012 & 19,0 \\
\hline 6-83 meses & Nacional & TADI & ELPI-2 & 2012 & $34,8^{*}$ \\
\hline 30-83 meses & Nacional & TVIP (LENGUAJE) & ELPI-2 & 2012 & 34,9 \\
\hline 0-72 meses & $\begin{array}{l}\text { Sector público } \\
\text { salud }\end{array}$ & PLS-IV (lenguaje) & $\begin{array}{l}\text { Evaluación de Impacto Nadie es Perfecto. } \\
\text { Línea base }\end{array}$ & 2012 & 30,0 \\
\hline 30-48 meses & $\begin{array}{l}\text { Sector público } \\
\text { salud }\end{array}$ & BATTELLE & $\begin{array}{l}\text { Evaluación efectividad del Programa de Apoyo } \\
\text { al Desarrollo Biopsicosocial. Cohorte tratada }\end{array}$ & 2013 & 33,8 \\
\hline
\end{tabular}

Fuente: Elaboración propia, con base a estudios. *Incluye normal con rezago.

'Fuentes:

1. Elaboración propia, con base a Encuesta Nacional de Calidad de Vida y Salud (2006) Ministerio de Salud de Chile.

2. Edwards M, Hurtado M, Bañados J, De Amesti A, Seguel X, Covarrubias M, Wormald A, Chadwick M. Qué efecto tiene asistir a Sala Cuna y Jardín Infantil desde los 3 meses hasta los 4 años de edad?: Estudio longitudinal en la Junta Nacional de Jardines InfantilesJUNJ. Psykhe 2012; 21: 87-104.

3. Centro de Microdatos-Universidad de Chile. (2010) Encuesta Longitudinal de Primera Infancia 1.

4. Centro de Microdatos-Universidad de Chile (2012) Encuesta Longitudinal de Primera Infancia 2

5. Bedregal, Carneiro, Cordero, Galasso y López (2012). Línea de base evaluación de impacto talleres "Nadie es Perfecto". Informe al Ministerio de Salud de Chile.

${ }^{2}$ Los instrumentos utilizados en estos estudios son:

1. Inventario de Desarrollo Battelle (Newborg, J. 2005. Battelle Developmental Inventory, 2nd edition. Rolling Meadows, IL: Riverside Publishing), que es una prueba diagnóstica de aplicación directa al niño. Se utilizan las normas norteamericanas.

2. La prueba de tamizaje "Desarrollo Infantil Temprano para Encuestas de Hogares" (DIEH) (Bedregal y cols, 2007 El desarrollo de un modelo para la evaluación de rezagos del desarrollo infantil de Chile. Rev Med Chile [online]. 2007,.135, n.3.), que se aplicó en la Encuesta de Calidad de Vida y Salud, en el módulo de infancia.

3. TVIP (Dunn, LI, Lugo DE, Padilla ER y Dunn LM. Test de Vocabulario en imágenes Peabody. American Guidance Service. Adaptación Hispanoamericana. Cacle Pines, MN: American Guidance Service. 1986.), corresponde a la prueba Peabody para lenguaje, ampliamente utilizada en Chile, en la versión adaptada para Hispanoamérica.

4. PLS-IV (Zimmerman IL, Steinerv V, Evatt Pond R. Preschool Language Scale, Fourth Edition. Spanish Edition. Pearson, 2002).

5. EEDP (Rodríguez S, Arancibia V, Undurraga C. Escala de evaluación de desarrollo psicomotor para niños entre 0 y 2 años. Santiago: Editorial Galdoc 1976), es una prueba de tamizaje, elaborada en Chile, en uso para niños menores de 2 años en el sector público de salud.

6. TADI (Edwards M, Gómez M, Pardo M. Test de Aprendizaje y Desarrollo Infantil. Documento de Trabajo. 2012), es una prueba chilena de valoración del desarrollo, en etapa de elaboración.

les de salud infantil orientados a la prevención de enfermedades, cuyo objetivo es identificar, tratar y prevenir de manera específica, cuyo principio base para la priorización es la costoefectividad, sin considerar los determinantes sociales y los derechos de las personas; y modelos neuro-maduracionales de desarrollo infantil temprano, cuyo objetivo es que el niño logre un hito funcional, determinado por una prueba estandarizada de valoración psicosocial del desarrollo, la que suele ignorar trayec- toria y contextos. Ambos enfoques han sido puestos en tela de juicio promoviéndose en la actualidad un modelo de atención de salud a la infancia, basado en un enfoque de derechos y de curso de vida.

El actual Programa de Salud de la Infancia con Enfoque Integral del Ministerio de Salud (2013), va en esa línea y es un aporte sustantivo al cambio de modelo. Pero hay que avanzar más. Algunos desafíos futuros para la política en salud infantil son: 
1. La promoción del desarrollo humano y derechos de la niñez, entendiendo que maximizar la salud de los niños y niñas es desarrollo, y maximizar el desarrollo psicosocial es salud, y que ambos (salud y desarrollo psicosocial son derechos). Este enfoque permite una mirada de curso de vida, y la incorporación decidida del enfoque de necesidades especiales de atención, y de inclusión social.

2. La identificación temprana de marcadores de enfermedades crónicas que alteran el curso de vida. Para esto es fundamental mantener una inteligencia activa en que concurran las sociedades científicas y las autoridades técnicas para generar un cuerpo de conocimiento vivo sobre epidemiología del curso de vida, que nutra a la toma de decisiones políticas.

3. La identificación temprana de vulnerabilidades y amenazas, e implementación de intervenciones para la modificación de trayectorias de desarrollo y de riesgos. Esto implica incorporar la mirada longitudinal del desarrollo psicosocial, integrada al desarrollo físico y el estado nutricional, con herramientas prácticas para los pediatras, y equipo a cargo del seguimiento de los niños y niñas, que permitan identificar las trayectorias. El diagnóstico se debería nutrir con la identificación de exposiciones a factores que alteren estas trayectorias y que se constituyen en amenazas externas al desarrollo integral (tanto de la familia, barrio, comuna, región, país), como también con el reconocimiento de las fortalezas y vulnerabilidades dadas por las características propias del niño/a. Esto implica comprender que el desarrollo integral NO es el resultado de una prueba de valoración del desarrollo sino que es la interpretación clínica de una prueba en un contexto de valoración de amenazas y vulnerabilidades, en la trayectoria de vida.

4. Las apertura de las intervenciones clínicas convencionales hacia las terapias complementarias y medicinas alternativas, de uso extendido en al menos un tercio de la población, y que no sólo amplían el arsenal terapéutico sino que apuntan a aspectos del desarrollo e interacción humana no consideradas necesariamente en la práctica habitual.

5. La generación de sistemas de apoyo a padres, comunidades, instituciones proveedoras de servicios para la niñez. Para esto se requiere avanzar en establecer competencias deseables (más que contenidos) para el apoyo en la parentalidad, el cuidado de la salud (de otros y la propia), la valoración $\mathrm{y}$ reconocimientos de derechos y deberes; que permitan formular estrategias educativas atingentes a las necesidades y perfiles.

6. Por otra parte, muchos países entendiendo la importancia del período peri-concepcional, han enfatizado el trabajo con adolescentes y jóvenes, en una lógica adicional de apoyo y promoción del proyecto de vida $\mathrm{y}$ parentalidad sana desde edades tempranas y reforzadas durante las diversas etapas del desarrollo humano.

7. Implementar un modelo de atención integrado verticalmente, en que los diversos niveles de complejidad de la atención se articulen con miras a objetivos comunes; e integración horizontal, que implica coordinaciones con otros sectores, sobre la base de una planificación común, con objetivos y metas claras sectoriales y compartidas. Esto implica una revisión de los modelos de gestión de servicios de salud, que tradicionalmente han estado basados en programas y no en sistemas integrados.

Hoy tenemos un desafío nuevo en salud infantil, que convoca nuevamente a todos los actores sociales, más allá de los pediatras (como lo fue durante la época de la sanidad y control de infecciones). Sin embargo, el rol de las sociedades científicas se hace cada vez más necesario para aportar con evidencias clínicas, sugerir modelos teóricos, y ser garantes y vigilantes del cumplimiento de los derechos de la niñez en las políticas de infancia.

\section{Referencias}

1.- UNICEF: Committing to Child Survival: A Promise Renewed Progress Report 2013. New York, UNICEF. 2013. 
2.- CEOC: Balance económico de Chile en el 2012. Santiago de Chile, Universidad de Talca. 2012.

3.- Ministerio de Desarrollo Social: Indicadores de pobreza, Encuesta de Caracterización de Hogares, 2011. http:// observatorio.ministeriodesarrollosocial.gob.cl/layout/ doc/casen/publicaciones/2011/pobreza_casen_2011.pdf

4.- Ministerio de Salud de Chile: Programa Nacional de Salud de la Infancia con Enfoque Integral. Santiago de Chile, Ministerio de Salud. Agosto 2013.

5.- Bedregal P: Diagnóstico y lineamientos para el mejoramiento de Chile Crece Contigo. Informe de Consultoría,
Banco Interamericano del Desarrollo, diciembre 2013.

6.- Vicente B, Saldivia S, De la Barra F, Melipillán R, Valdivia M, Kohn R: Salud mental infanto-juvenil en Chile y brechas de atención sanitarias. Rev Med Chile 2012; 140: 447-57.

7.- Bedregal P, Carneiro P, Cordero M, Galasso E, López I: Evaluación de Impacto del programa "Nadie es Perfecto". Resultados pre-tratamiento. Informe al Ministerio de Salud de Chile, abril 2013.

8.- UNICEF: Cuarto estudio de maltrato infantil en Chile. Santiago, UNICEF. 2012. 